\title{
Coupling factors, visual rhythms, and synchronization ratios
}

\author{
UDO WILL \\ Cognitive Ethnomusicology, School of Music, Ohio State University
}

\begin{abstract}
The inter-group entrainment study by Lucas, Clayton, and Leante (2011) is an interesting research report that advances studies in both empirical ethnomusicology and entrainment research in several ways, and provides an important addition to the much needed empirical case studies on musical entrainment. I submit that the authors' analysis of an instant of resistance to entrainment is a key demonstration of the complementarity of analytical and ethnographic approaches in entrainment research. Further, I suggest that the evidence for the influence of visual information on entrainment supports the idea that there are two types of visuo-temporal information, each with different influence on the entrainment process, those derived from static and those from moving visual objects. As a final point, I argue that if we take into consideration the possibility of higher-order synchronization, some of the authors' interpretations would need modification.
\end{abstract}

Submitted 2011 December 31; accepted 2012 January 29.

KEYWORDS: analytical studies, coupling factors, auditory rhythms, visual rhythms, limb movements, synchrogram, higher-order synchronization.

I enjoyed reading this study on inter-group entrainment by Lucas, Clayton and Leante (2011), which is an interesting research report that advances studies in both empirical ethnomusicology and entrainment research in several ways. It provides an attractive new case to the much needed empirical studies on musical entrainment. My comments address issues related to the complementarity of ethnographic and analytic approaches, the interaction of visual and auditory information in musical entrainment, and synchronization ratios.

\section{ENTRAINMENT AND COUPLING}

The inter-group entrainment study by Lucas et al. (2011) is a stimulating demonstration of the status and scope of (performance) analytical studies in ethnomusicological research where socio-cultural factors often cannot be subjected to experimental manipulations without severe disruptions of the performance context. Clearly, as the study shows, analytical studies are not just 'preliminary' or 'pre-experimental', only performed to help formulating initial hypotheses; rather they are an important means - often the only available ones - to test hypotheses, interpretations, and assumptions in an ecologically valid manner that is difficult, if not impossible, to achieve in experimental studies in the lab. They have to be considered an essential complement to experimental studies. Their data and results frequently lead to further research that requires experimental approaches, and they also permit the testing of hypotheses derived from experimental studies (Will, 2011).

The Lucas et al. (2011) paper also highlights the importance of a close collaboration of ethnographic and analytical approaches in entrainment research. The need for this collaboration arises from the fact that entrainment theory predefines neither the nature of the implemented oscillatory processes nor the nature of the interaction (coupling) between these processes - they have to be identified by the applying disciplines: what acts as coupling forces and the kind and degree of interactions is dependent on the specific systems in which the oscillatory processes are implemented. The workings of this collaboration are clearly demonstrated when the authors introduce the concept of 'resisting entrainment', referring to the phenomenon that group identity, or much more the intent to demonstrate it, is identified as a factor that influences how participating groups entrain to each other. 
Human temporal processing is known to be influenced by physiological as well as cognitive factors like knowledge and experience (e.g. Block, 1990), and these factors also seem to affect entrainment. McAuley, Jones, Holub, Johnston, and Miller (2006) have shown that biological factors like age can act as regulators of coupling strength. Our bodily responses to music are also influenced by familiarity with the music, by musical training, cultural practices, and even belief systems (see Will \& Turow, 2011, for examples and references), and, as Lucas et al. remind us, Kelso (1995) suggested that intention might be another factor "acting on or perturbing the basic (intrinsic) coordination dynamics". In their current study the authors present a related factor, group identity, expressed as rhythmic identity or rhythmic distinctness between the participating groups, and identify it as kind of 'negative coupling factor': the stronger a group's identity or, in this case, their musical competence, the better it is able to maintain its identity (rhythmic distinctness) in a spatially close encounter with another group. By bringing to bear the available ethnographic information the analyses of the four examples show that it is not so much group identity as such that acts as a coupling factor, but the specific function that group identity has in specific performance situations that determines whether this factor acts as a (negative) coupling factor or not: in the current study it is only when the groups in an encounter are from different communities (extracts 3 and 4) that there is an intent to mark their respective group identity and this intent then influences the coupling between the groups.

With this analysis the authors contribute to an improved understanding of the complex nature of coupling. Interactions between oscillatory processes are not only influenced by biological or physiological factors relating to how these processes are implemented, they are also shaped by cognitive and emotional factors. The presented case study therefore effectively highlights the special status of the concept of coupling, linking physiological, psychological and socio-cultural domains within the framework of entrainment theory.

\section{ENTRAINMENT AND THE ROLE OF VISUAL RHYTHMS}

The study of Lucas et al. (2011) also draws attention to the significance of visual rhythms in entrainment, which seems in line, as the authors remark, with other studies that "have pointed to the role of visual information in unintentional interpersonal coupling (Richardson, Marsh, \& Schmidt, 2005; Richardson, Marsh, Isenhower, Goodman, \& Schmidt, 2007; Schmidt \& O’Brien, 1997)” (p. 77). However, a couple of experimental studies indicate that claims about the role of visual information in entrainment are not uncontested. On the one hand, Wada et al. (2003) showed that when ambiguous auditory temporal cues were presented, the change in the frequency of the visual stimuli was associated with a perceived change in the frequency of the auditory stimuli, and Guttman, Gilroy, and Blake (2005) have suggested that for temporal tasks visual rhythms are automatically and obligatorily encoded in the auditory domain. On the other hand, a study by McAuley and Henry (2010) indicates that there is, at least for beat-based temporal judgments, a significantly greater sensitivity for auditory rhythms than for visual rhythms, and Patel, Iversen, Chen, and Repp (2005) reported for a multimodal beat perception experiment that rhythmic visual sequences "do not easily induce a beat on their own, let alone a multi-level metrical structure". I submit that this apparent contradiction may find an explanation if we take account of the different stimuli applied in these studies. A case can be made that the results of McAuley and Henry (2010) and Patel et al. (2005) are due to the specific stimuli used: in both studies the stimuli were visual flashes. It can be argued that the use of visual movement stimuli would lead to different results because trajectories and movements can be followed visually and therefore create higher expectancies and augmented predictability - stimulus features that have a marked effect on entrainment.

This argument finds support from behavioral, imaging and physiological studies. An improvement of visuomotor synchronization by motion stimuli has been demonstrated by Hove, Spivey, and Krumhansl (2010), who directly compared effects of flashing and moving visual stimuli. Other studies indicate the importance of body and limb movements in synchronization and entrainment: In an analytical study on Hindustani classical music, in which performers' arm and hand gestures occupy a special place, it was shown that these movements influence the temporal behavior of other performers as well as that of the audience (Clayton, 2007). In an experimental study on pulse synchronization to a vocal alap performance we found that there was significant higher between-subject agreement in tapping to the audio-visual than to the audio-only presentation (Will, Utter, Clayton, \& Leante, 2005). These results suggest that visual 
rhythms of limb (and body) movements may well be able to disambiguate the auditory rhythmic structure of a performance and shape pulse induction in listeners/observers.

The special status of temporal information related to limb and body movements is also indicated by several imaging studies showing that motor areas of the human brain are activated during visual movement perception. For example, Schubotz, Friederici, and vonCramon (2000), using visual quasimovement stimuli without eliciting subjects' motor responses, found that supplementary motor area, premotor cortex, and primary motor cortex (as well as Basal Ganglia, Cerebellum, etc.) were involved in a rhythm monitoring task. Naito (2004) argues that the link between limb movement perception and motor areas probably derives from the fact that sensory input from and about limb movements is essential for fine-grained control over these movements. In addition, Schroeder, Lakatos, Kajikawa, Partan, and Puce (2008) demonstrate that non-auditory inputs to the primary auditory cortex can modulate on-going neuronal activity in a way that amplifies appropriately timed auditory inputs. They hypothesize that oscillations in the auditory cortex maybe 'predictively' modulated by visual input, so that related auditory input arrives during a high excitability phase and is thus amplified. Together, these findings seem to suggest that either the highly predictable visual rhythms of limb movements are more effective at influencing auditory temporal information than those of visually stationary objects (flashing dots, etc.), or temporal information from limb movements is processed differently from that of non-body related objects. This latter option seems to be part of the system(s) of human time measurement proposed by Lewis and Miall (2003), who argue that there may be more than one timing system, one of which being connected to the motor system and dealing with movement related time processing and stimuli with predictability: "Taken together with the suggestion that motor circuitry can be used to measure temporal intervals even in the absence of movement, these two proposals suggest the preferential use of motor circuitry for continuous, predictable (as opposed to discontinuous or unpredictable) time measurement tasks" (Lewis \& Miall, 2003).

The empirical evidence forwarded in the current paper by Lucas et al. (2011) lends additional support to the idea that it is visual information related to movements of body and/or limbs that forms an important and effective component of musical entrainment. As the new evidence fits well into current models of multiple timing systems, it is bound to stimulate further studies, analytical as well as experimental ones, to explore the underlying mechanisms and to improve our understanding of the interaction between visual and auditory rhythms in entrainment.

\section{HIGHER-ORDER SYNCHRONIZATION}

My final comment addresses aspects of the synchrogram analysis (i.e. analysis of visual display of phase relationship between oscillatory processes) employed in the present study. A potential limit of analytical studies on entrainment is the availability of variables which permit the determination of whether identified synchronization is indeed due to entrainment. Entrainment implies interaction of the rhythmic processes involved, whereas synchronization can also occur, at least intermittently, between oscillators that are not interacting (Kreuz, 2004). Fortunately, live performances frequently contain various 'perturbations' that allow assessing the presence of entrainment, as for example the change of rhythmic pattern in case study 3 of Clayton, Sager, and Will (2005). In the present study, Lucas et al. (2011) explore another type of 'perturbation', that of tempo changes: "A correlation between two tempo trends can be regarded as a series of perturbations and reactions: in the simplest case, one group spontaneously speeds up and the other responds. Correlated averaged time series therefore provide strong evidence for entrainment." (p. 80). Interestingly, their examples also seem to include a case where entrainment is indicated by the presence of a stationary phase relationship occurring in conjunction with non-correlated tempi. Identification of this case, however, hinges on the recognition of the phenomenon of higher-order synchronization, and the current study, like most research on musical entrainment, seems to ignore this possibility (all their analyzed cases deal with entrainment/synchronization at 1:1 ratios), although higher-order synchronization (synchronization at ratios $\mathrm{n}: \mathrm{m}$, where $\mathrm{n} \neq \mathrm{m}$ and $\mathrm{n}, \mathrm{m} \geq 2$ ) is an established phenomenon in dynamical systems (Pikovsky, Rosenblum, \& Kurths 2001), especially in human physiology (Schäfer, Rosenblum, Abel, \& Kurths, 1999). Once this option is considered, some interesting alternative and/or modified interpretations of the reported results emerge. 
This can be shown by taking a look at the third example (extract 3), of which the authors write: "...the JC group maintains a slightly higher tempo $(117-124 \mathrm{bpm})$ than the JIC (108-104 bpm), ruling out any possibility of period and phase locking." (p. 90), and they see this supported by the corresponding relative phase time plot (their Figure 20), which "shows no stabilization". However, there is an alternative interpretation of this synchrogram that is not explored in the paper: The tempo plot for this example (their Figure 19) indicates that the two groups maintain a relative constant tempo relationship in the first part of the analyzed section, and the corresponding phase plot (their Figure 20) shows a horizontal layering that segregates into a limited number of parallel bands of phase values at around the 180 secs mark, suggesting a higher-order synchronization. If this can be substantiated, e.g. through an analysis of the corresponding phase density histograms, 'resisting entrainment' may have to be redefined as 'resisting synchronization at a 1:1 ratio', and it would have to allow for a, perhaps unavoidable, synchronization at higher levels; after all, the analyzed section spans nearly three minutes, and it may be extremely difficult for two groups playing in proximity to remain completely unaffected by each other for any extended amount of time.

Taking higher-order synchronization into consideration may also influence the interpretation of sections where synchronization cannot be identified unambiguously. This can be shown by discussing extract 2 in Lucas et al. (2011), which contains the above mentioned case of a stable phase relationship cooccurring with changing tempo relationships. In this example, Lucas et al. identify two synchronized and entrained periods, between 58-95 secs and 720-840 secs, respectively. While the identification of the later period seems unproblematic, identification of the first one raises certain questions, as the authors acknowledge: "if the two rhythms become entrained, the apex of the arch would be expected to flatten as the relative phase stabilizes. Visual evidence of the latter is weak (Figure 13), although the chart is consistent with the hypothesis that the groups mutually entrain for a short period when their tempos almost match and their relative phase reaches $0^{\circ} . "($ p. 86). The problem is that this first period as a whole shows significant correlation, but the sub periods (55-75 and 75-95) do not. Interestingly the phase plot clearly shows a horizontally layered structure starting at ca. 120 secs that slowly dissolves at around 360 secs. This layering, again, indicates higher-order synchronization and can be interpreted as follows: With the tempo change shown by the Arturos Moçambique (AM) group (at $155 \mathrm{secs}$ ), after starting a new song, there seems to be higher-order phase stabilization lasting for over a minute. It subsequently dissolves and at the second tempo jump of AM the synchronization is lost. The higher-order phase relationship at 155 secs may be an indicator that the tempo at this point has not been selected arbitrarily but may instead be related to the tempo attained before the jump; unfortunately, we cannot be certain about this as there are some missing data at the start of the tempo jump. However this may be, the remarkable point here is that higher order stabilization seems to be present over an extended amount of time during which the tempo relationship between the groups is changing constantly (AC remains relatively constant while AM is accelerating). This would be another strong argument for entrainment during the first part of extract 2.

There are indications of further instances of higher-order synchronization (e.g. at the beginning of the $1^{\text {st }}$ example and at the end of the $2^{\text {nd }}$ example) but available data are not complete and it is therefore not possible to draw any conclusions from them. Nevertheless, their multiple occurrence in the present study indicates that they may be a rather frequent phenomenon, and further explorations of their significance in performances seem appropriate to gain better insight into the full spectrum of musical entrainment phenomena.

\section{REFERENCES}

Block, R. A. (1990). Models of psychological time. In R. A. Block (Ed.), Cognitive Models of Psychological Time. Hillsdale, NJ: Erlbaum, pp. 1-35.

Clayton, M. R., Sager, R., \& Will, U. (2005). In time with the music: The concept of entrainment and its significance for ethnomusicology. European Meetings in Ethnomusicology, Vol. 11, pp. 3-75.

Clayton, M. R. L. (2007). Observing entrainment in Indian music performance: Video-based observational analysis of tanpura playing and beat marking. Musicae Scientiae, Vol. 11, No. 1, pp. 27-60. 
Guttman, S. E., Gilroy, L. A., \& Blake, R. (2005). Hearing what the eyes see: Auditory encoding of visual temporal sequences. Psychological Science, Vol. 16, pp. 228-235.

Hove, M. J., Spivey, M. J., \& Krumhansl, C. L. (2010). Compatibility of motion facilitates visuomotor synchronization. Journal of Experimental Psychology: Human Perception and Performance, Vol. 36, No. 6, pp. 1525-1534.

Kelso, J. A. S. (1995). Dynamic Patterns: The Self-Organization of Brain and Behavior. Cambridge, Mass.: MIT Press.

Kreuz, T. (2004). Measuring Synchronization in Model Systems and Electroencephalographic Time Series from Epilepsy Patients. NIC series, Vol.21. Jülich: John von Neumann Institute for Computing.

Lewis, P. A., \& Miall, R. C. (2003). Distinct systems for automatic and cognitively controlled time measurements: evidence from neuroimaging. Current Opinion in Neurobiology, Vol. 13, pp. 250-255.

Lucas, L., Clayton, M., \& Leante, L. (2011). Inter-group entrainment in Afro-Brazilian Congado ritual. Empirical Musicology Review, Vol. 6, No. 2, pp. 75-102.

McAuley, J. D., Jones, M. R., Holub, S., Johnston, H. M., \& Miller, N. S. (2006). The time of our lives: Life span development of timing and event tracking. Journal of Experimental Psychology: General, Vol. 135, pp. 348-367.

McAuley, J. D., \& Henry, M. J., (2010). Modality effects in rhythm processing: auditory encoding of visual rhythms is neither obligatory nor automatic. Attention, Perception, \& Psychophysics. Vol. 72, pp. 13771389 .

Naito, E. (2004). Sensing limb movements in the motor cortex: How humans sense limb movements. The Neuroscientist, Vol. 10, No. 1, pp. 73-82.

Patel, A. D., Iversen, J. R., Chen, Y., \& Repp, B. H. (2005). The influence of metricality and modality on synchronization with a beat. Experimental Brain Research, Vol. 163, pp. 226-238.

Pikovsky, A. S., Rosenblum, M. G., \& Kurths, J. (2001). Synchronization. A universal concept in nonlinear sciences, Cambridge, UK.: Cambridge University Press.

Richardson, M. J., Marsh, K. L., Isenhower, R. W., Goodman, J. R. L., \& Schmidt, R. C. (2007). Rocking together: Dynamics of intentional and unintentional interpersonal coordination. Human Movement Science, Vol. 26, pp. 867-891.

Richardson, M. J., Marsh, K. L., \& Schimdt, R. C. (2005). Effects of visual and verbal interaction on unintentional interpersonal coordination. Journal of Experimental Psychology: Human Perception and Performance, Vol. 31, No. 1, pp. 62-79.

Schäfer, C., Rosenblum, M. G., Abel, H. H., \& Kurths, J. (1999). Synchronization in the human cardiorespiratory system. Physical Review E, Vol. 60, pp. 857-870.

Schmidt, R. C., \& O'Brien, B. (1997). Evaluating the dynamics of unintended interpersonal coordination. Ecological Psychology, Vol. 9, No. 3, pp. 189-206.

Schroeder, C.E., Lakatos, P., Kajikawa, Y., Partan, S., \& Puce, A. (2008). Neuronal oscillations and visual amplification of speech. Trends in Cognitive Sciences, Vol. 12, No. 3, pp. 106-113.

Schubotz,R. I., Friederici, A. D., \& vonCramon, D. Y. (2000). Time perception and motor timing: A common cortical and subcortical basis revealed by fMRI. NeuroImage, Vol. 11, pp. 1-12. 
Wada, Y., Kitagawa, N., \& Noguchi, K. (2003). Audio-visual integration in temporal perception. International Journal of Psychophysiology, Vol. 50, pp. 117-124.

Will. U. (2011). Perspektiven einer Neuorientierung in der Kognitiven Musikethnologie [Prospects for a reorientation in cognitive ethnomusicology]. In: W. Steinbeck (Ed.), Selbstreflexion in der Musikwissenschaft. Kölner Beiträge zur Musikwissenschaft, Vol. 16. Kassel: Bosse Verlag, pp. 193-211.

Will, U. \& Turow, G. (2011). Introduction to entrainment and cognitive ethnomusicology. In: G. Turow \& J. Berger (Eds), Music, Science and the Rhythmic Brain. Cultural and Clinical Implications. New York: Routledge, pp. 3-30.

Will, U., Utter, H., Clayton, M., \& Leante, L. (2005): Influence of auditory and visual information on the tapping responses to vocal alap. Paper presented at the Entrainment Network Conference, The Ohio State University, Columbus. 5 May, 2005. 